\title{
A Rho-kinase inhibitor improves cardiac function after 24-hour heart preservation
}

Mariko Kobayashi, MD, Yoshihisa Tanoue, MD, Masataka Eto, MD, Hironori Baba, MD, Satoshi Kimura, MD, Shinichiro Oda, MD, Kenichiro Taniguchi, MD, and Ryuji Tominaga, MD

Objective: The Rho-kinase signaling pathway is associated with coronary vasculopathy and myocardial dysfunction after cardiac transplantation. This study evaluated whether using a Rho-kinase inhibitor during allograft storage could limit early endothelial dysfunction and improve myocardial performance after reperfusion.

\begin{abstract}
Methods: This experiment was performed with an isolated working rabbit heart model and a support rabbit. Donor hearts (control group, $\mathrm{n}=8$ ) were arrested with an extracellular type of cardioplegia, preserved with University of Wisconsin solution, and then immersed in University of Wisconsin solution for 24 hours $\left(1^{\circ} \mathrm{C}\right)$. The Rho-kinase inhibitor (Rho-kinase inhibitor group, $\mathrm{n}=8$ ) was administrated in the cardioplegic solution, the preservation University of Wisconsin solution, and the storage University of Wisconsin solution. Left ventricular performance was evaluated from the modified Frank-Starling curve in the working mode. Coronary blood flow and donor heart rate were measured in Langendorff mode. Effective evaluation of the Rho-kinase inhibitor was inferred from phosphorylated myosin light chain. The expression of endothelial nitric oxide synthase mRNA was analyzed to assess endothelial function.
\end{abstract}

Results: The Frank-Starling curve showed a significant left and upward shift in the Rho-kinase inhibitor group compared with the control group $(P<.05)$. The coronary blood flow and heart rate in the Rho-kinase inhibitor group at 120 minutes was significantly higher than in the control group $(P<.05)$. Phosphorylated myosin light chain was significantly suppressed in the Rho-kinase inhibitor group $(P<.05)$. Endothelial nitric oxide synthase mRNA levels in the Rho-kinase inhibitor group increased 4-fold relative to those seen in the control group.

Conclusions: Treatment with Rho-kinase inhibitor during allograft harvest and storage enhanced coronary blood flow and ventricular recovery through nitric oxide-dependent endothelial protection after reperfusion. Rhokinase inhibitor could help prevent early myocardial dysfunction after transplantation.

In clinical heart transplantation prolonged ischemia of cardiac allografts is statistically associated with poor postoperative outcomes, ${ }^{1}$ especially if the donor is elderly. ${ }^{2}$ Early myocardial dysfunction related to prolonged cardiac arrest is caused by severe ischemia-reperfusion injury, which involves damage to the cardiomyocytes, vascular smooth muscle, and endothelial cells through intracellular calcium overload, infiltration of inflammatory cells, and the production of free radicals and cytokines. Although the mechanism of ischemia-reperfusion injury is extremely complex, cardiac allograft protection by preventing ischemia-reperfusion injury would ameliorate early and long-term negative outcomes, be feasible for prolonged ischemia, and expand the donor pool.

The small guanosine triphosphate binding protein, Rho, and its downstream effecter, Rho-kinase, are involved in signal transduction initiated by agonists such as angiotensin II,

From the Department of Cardiovascular Surgery, Kyushu University, Fukuoka, Japan. This work was supported in part by grants from the Japanese Ministry of Education, Science, Sports, Culture, and Technology, Tokyo, Japan (no. 19790975).

Received for publication May 3, 2008; revisions received July 9, 2008; accepted for publication July 26, 2008.

Address for reprints: Yoshihisa Tanoue, MD, Department of Cardiovascular Surgery, Kyushu University, 3-1-1 Maidashi Higashi-ku Fukuoka, 812-8582, Japan (E-mail: tanoue@heart.med.kyushu-u.ac.jp).

J Thorac Cardiovasc Surg 2008;136:1586-92

$0022-5223 / \$ 34.00$

Copyright (c) 2008 by The American Association for Thoracic Surgery doi:10.1016/j.jtcvs.2008.07.038 thrombin, endothelin I, norepinephrine, and urotensin in both endothelium and vascular smooth muscle. ${ }^{3}$ The Rhokinase signaling pathway plays an important role in vascular diseases, including hypertension, myocardial and cerebral infarction, vascular spasm, atherosclerosis, and ischemia-reperfusion injury. ${ }^{3-5}$ The pathogenic cellular upregulation of Rho and Rho-kinase affects actin cytoskeleton organization, cell adhesion and migration, and apoptosis; increased monocyte chemoattractant protein 1 and plasminogen activator inhibitor 1 levels; and reduced endothelial nitric oxide synthase (eNOS) levels. ${ }^{3,6-8}$ Rho-kinase inhibitors (RKIs) can ameliorate various pathogenic vascular disorders in both animals and human subjects. ${ }^{4,9-10}$

The direct influence of a RKI, fasudil hydrochloride, in prolonged organ preservation has not been defined. To develop appropriate preservation and prevent ischemia-reperfusion injury, we supplemented cardioplegic and preservation solutions with RKI and evaluated functional recovery using an isolated working rabbit heart model with a support rabbit. ${ }^{11-13}$ We hypothesized that adding RKI to both cardioplegic and preservation solutions would provide both endothelial and myocardial preservation after reperfusion.

\section{MATERIALS AND METHODS}

\section{Animal Care}

The experiments were reviewed by the Ethics of Animal Experiments Committee of the Faculty of Medicine, Kyushu University, and performed 


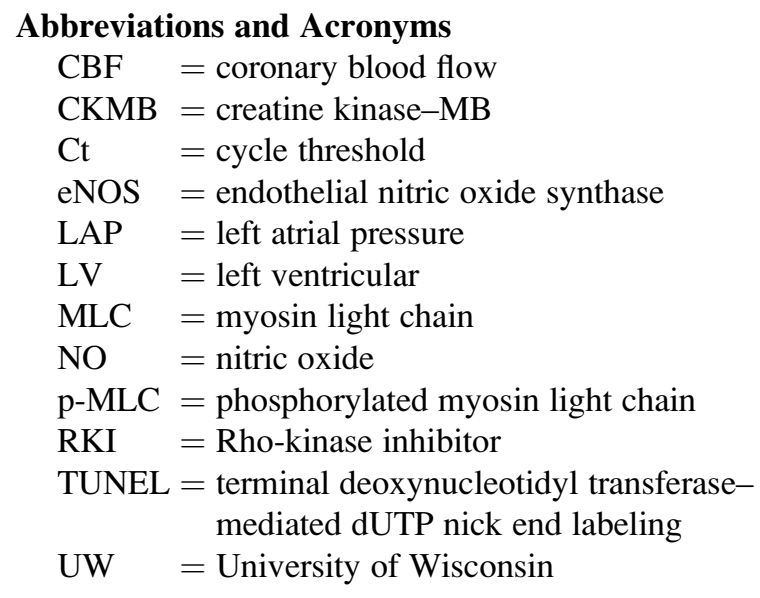

under the "Guidelines for animal experiments" of the Faculty of Medicine, Kyushu University, Legal Ruling (no. 105) and Notification (no. 6) of the Japanese Government.

\section{Animal Preparation}

Forty male Japanese white rabbits ( 16 donor rabbits, 16 support rabbits, and 8 blood donors) weighing 3.0 to $3.3 \mathrm{~kg}$ were used. General anesthesia was induced with sodium thiamylal $(20 \mathrm{mg} / \mathrm{kg}$ ) through the dorsal marginal ear vein, and then a tracheal tube was inserted through the mouth and connected to a mechanical ventilator with $100 \%$ oxygen. Additional vecuronium bromide $(1 \mathrm{mg} / \mathrm{kg})$ and fentanyl citrate $(30 \mu \mathrm{g} / \mathrm{kg})$ were intravenously administered. The femoral arterial pressure of both donor and support rabbits was monitored continuously. This study was performed with an isolated working rabbit heart model using support rabbit blood, as described in detail by Kajihara and colleagues. ${ }^{11-13}$

\section{Donor Heart Management}

The donor rabbit heart and aortic arch were exposed through a median sternotomy. After heparinization ( $300 \mathrm{U} / \mathrm{kg}$ ), the innominate artery was cannulated to administer the cardioplegic solution. The inferior vena cava was transected to decompress the heart, and then the aortic arch was crossclamped. In the control group $(n=8)$ cardiac arrest was induced with an infusion of $10 \mathrm{~mL} / \mathrm{kg}$ extracellular-type Kyushu University cardioplegic solution, ${ }^{13,14}$ and then $20 \mathrm{~mL} / \mathrm{kg}$ University of Wisconsin (UW) preservation solution was infused to wash out the coronary vascular cardioplegia. The excised heart was immersed in UW solution $(55 \mathrm{~mL})$ for 24 hours. The temperature and administration velocity of both cardioplegic and UW solutions were maintained at $4{ }^{\circ} \mathrm{C}$ and $12 \mathrm{~mL} / \mathrm{min}$, respectively. In the RKI group $0.1 \mathrm{mg} / \mathrm{kg}$ RKI was administered with the cardioplegia and UW solutions, and $0.5 \mathrm{mg} / \mathrm{kg}$ RKI was added to the immersion UW solution. All excised hearts were preserved at $1{ }^{\circ} \mathrm{C}$ by using heat exchange for 24 hours, followed by 120 minutes of reperfusion.

\section{Support Rabbit and Cross-circulation System}

After induction of general anesthesia and heparinization (300 U/kg), the common carotid arteries and external jugular veins of support rabbits were exposed and cannulated. Fentanyl citrate $(100 \mu \mathrm{g} / \mathrm{h})$, vecuronium bromide $(1 \mathrm{mg} / \mathrm{h})$, and heparin $(1000 \mathrm{U} / \mathrm{h})$ were used to maintain anesthesia and prevent coagulation. Oxygenated blood from the common carotid artery of the support rabbit was introduced to a cannula connected to the ascending aorta of the donor heart with a microtube pump. Blood draining from the system was returned to the jugular vein through another microtube pump. The pulmonary vein and vena cava of the donor heart were closed, and a double- lumen cannula was inserted into the left atrium. One lumen was connected to a pressure transducer to measure left atrial pressure (LAP). The other lumen was connected to an atrial reservoir (preload). During systemic circulation, hematocrit levels in the perfusion blood were maintained at more than $23 \%$, and blood temperature was maintained at $37^{\circ} \mathrm{C}$. Arterial perfusion blood gas and $\mathrm{pH}$ were normalized by changing the settings of the mechanical ventilator and using sodium bicarbonate. The hemodynamics of the support rabbit were stabilized by maintaining a systolic blood pressure of greater than $90 \mathrm{~mm} \mathrm{Hg}$. At the end of the study, the donor heart was cut into 3 transverse slices, and pieces of each slice were stored at $-80^{\circ} \mathrm{C}$. The remaining block was fixed in $10 \%$ formaldehyde.

\section{Modified Frank-Starling Curve}

The donor heart was perfused and stabilized with constant coronary perfusion at $60 \mathrm{~mm} \mathrm{Hg}$ in Langendorff mode for 60 minutes, and then the working mode was started. In working mode the heart was paced atrially at 250 beats/min, and the aortic afterload pressure was fixed at $60 \mathrm{~mm} \mathrm{Hg}$. LAP was gradually increased by raising the atrial reservoir, and aortic flow rates were measured at each level of LAP. Based on these data, we constructed a modified Frank-Starling curve. After measurement of left ventricular (LV) function, the working mode was reversed to Langendorff mode, and at 120 minutes after reperfusion, LV function was evaluated in working mode again.

\section{Coronary Blood Flow and Heart Rate}

The maximum coronary blood flow $(\mathrm{CBF})$ and heart rate of the donor heart were measured in Langendorff mode at 10, 60, and 120 minutes after reperfusion.

\section{Measurement of Creatine Kinase-MB}

Serum creatine kinase-MB (CKMB) levels were measured with a chemiluminescent immunoassay (SRL, Inc, Tokyo, Japan). The change of CKMB level between 60 and 120 minutes is presented, with the level at 60 minutes representing $100 \%$.

\section{Quantitative Real-time Reverse Transcriptase- Polymerase Chain Reaction}

The gene expression of eNOS in myocardium was measured by means of quantitative real-time reverse transcriptase-polymerase chain reaction performed according to the recommendations of the Premix Ex Taq-Perfect Real-Time protocol (Takara Bio, Inc, Shiga, Japan). Total RNA was isolated from homogenized myocardium with ISOGEN (Nippon Gene Co Ltd, Toyama, Japan). The PrimeScript first strand cDNA (Takara Bio, Inc) was made with random primers to reverse transcribe RNA to cDNA. The eNOS probe and primers were designed based on the published sequence of rabbit eNOS (GenBank accession no. AY964103) ${ }^{15}$ and were produced by Applied Biosystems Japan Ltd. The amount of target cDNA was quantified on a Sequence Detection System ABI 7000 (Applied Biosystems Ltd, Foster City, Calif) by using rabbit eNOS-specific primers and probe. Relative gene expression was calculated by using the $\delta \delta$ cycle threshold $(\mathrm{Ct})$ method. Relative eNOS levels were determined by normalizing the $\mathrm{Ct}$ value to the rabbit glyceraldehyde-3-phosphate dehydrogenase signal measured from the same sample. The sample with lowest eNOS expression was arbitrarily selected as a calibrator value, and the other quantities are expressed as an $\mathrm{n}$-fold difference relative to this calibrator.

\section{Western Blot Analysis for Phosphorylated Myosin Light Chain}

The extent of phosphorylation of the 20-kd myosin light chain (MLC) in myocardium was quantified by means of Western blot analysis, as previously described, to evaluate Rho-kinase activity. ${ }^{16}$ Protein samples were separated by means of SDS-PAGE with $15 \%$ Tris-HCL gels (Bio-Rad 
Laboratories, Inc, Tokyo, Japan). Twenty microliters of each sample containing $50 \mu \mathrm{g}$ of protein was applied to the gel and run for 90 minutes at $100 \mathrm{~V}$. Separated proteins were transferred to a membrane at $100 \mathrm{~V}$ for 60 minutes. The membrane was incubated with an anti-mouse monoclonal phosphorylated-MLC (p-MLC) antibody (Cell Signaling Technology, Inc, Danvers, Mass), followed by incubation with an anti-mouse IgG horseradish peroxidase-labeled secondary antibody. The region containing MLC was detected with a monoclonal anti-mouse MLC primary antibody (Sigma-Aldrich, Inc, St Louis, Mo) and an anti-mouse IgM horseradish peroxidase-labeled secondary antibody. After detection was performed with a luminoimage analyzer, the intensity of both the unphosphorylated and phosphorylated MLC bands was quantified with National Institutes of Health Image $\mathbf{J}$ software. The percentage of the phosphorylated form was measured to express the extent of the MLC phosphorylation. HEK293 cells were used as a positive control for both specific primary antibodies.

\section{Histology in the Myocardium}

Cardiac tissue was fixed in 10\% formaldehyde, dehydrated, and embedded in paraffin. Each block was cut into $5-\mu$ m-thick slices and stained with hematoxylin and eosin to evaluate infiltrating cells.

Apoptotic myocytes were detected with a terminal deoxynucleotidyl transferase-mediated dUTP nick end labeling (TUNEL) assay by using a Mebstain Apoptosis Kit II (Medical and Biological Laboratories Co, Ltd, Nagoya, Japan), according to the manufacturer's instructions. Myer's hematoxylin was used as the counterstain. The ratio of TUNEL-positive nuclei per 1000 total nuclei was measured.

\section{Statistical Analysis}

SPSS statistical software for Windows (version 15.0; SPSS, Inc, Chicago, Ill) was used for data analysis. Data are expressed as the mean value \pm standard error of the mean. Statistical comparisons were performed by using unpaired Student $t$ tests. The relationship between LAP and aortic flow was analyzed by using a multiple regression model with a dummy variable technique to investigate intergroup differences.

\section{RESULTS}

\section{Modified Frank-Starling Curve}

Modified Frank-Starling curves were obtained from each group at both 60 and 120 minutes after reperfusion, and the equations obtained from multiple regression analyses are shown in Figure 1. The values of the $\mathrm{x}$-intercepts and the slopes at the $\mathrm{x}$-intercepts obtained from regression curves in each group are shown in Table 1 . The value of the $x$-intercept in the RKI group was significantly higher than that in the control group, indicating better function in the control group than in the RKI group. The value of the slope at the $\mathrm{X}$-intercept obtained from individual regressions in the control group was significantly lower than in the RKI group, indicating better function in the control group than the RKI group.

There was no difference between 60 and 120 minutes in the RKI group $(P=.13)$, but there was a difference in the control group $(P=.04)$.

\section{CBF and Heart Rate}

The maximum CBF at 10, 60, and 120 minutes after reperfusion in each group is shown in Figure 2, A. The CBF in the RKI group at 10 and 120 minutes was significantly higher than in the control group $(P<.05)$. The heart rate of the donor after reperfusion was more similar to the normal rabbit rate in the RKI group than in the control group (Figure $2, B)$.

\section{Serum Levels of CKMB}

Serum CKMB levels in the coronary effluent at 10 and 120 minutes were not significantly different between the control and RKI groups. However, the CKMB change from 60 to 120 minutes was lower in the RKI group than in the control group $(P<.05$; Figure $3, A)$.

\section{eNOS Synthesis}

The relative amount of eNOS mRNA was quantified by means of linear extrapolation of the Ct values, and eNOS mRNA levels in the RKI group were 4-fold greater than those in the control group (Figure 3, $B$ ).

\section{Suppression of MLC Phosphorylation by RKI}

RKI treatment significantly blocked the extent of MLC phosphorylation (the phosphorylated form as a percentage of total MLC) at 2 hours after reperfusion $(P<.05$; Figure $3, C)$.

\section{Pathologic Findings}

There was no obvious change in myocardial or vascular structure in the control and RKI groups, and infiltrating cells were not observed. TUNEL-positive nuclei were detected in $0.3 \%$ per 1000 nuclei in the control group and $0.1 \%$ per 1000 nuclei in the RKI group $(P=.4)$.

\section{DISCUSSION}

We found that inhibition of Rho-kinase during allograft harvest and storage markedly improved recovery of myocardial function. The improved myocardial function seemed to result from heightened preservation of endothelium and vascular smooth muscle. This study clearly showed the cardioprotective effect of RKI during cardiac allograft storage in the isolated working rabbit heart model with a support rabbit.

This experimental model offers major advantages, in particular by demonstrating pharmacologic effects at the organ level. Use of a working mode and a support rabbit provided a closer approach to in vivo conditions. Perfusate containing erythrocytes, leukocytes, platelets, and inflammatory cytokines is more commonly compared with crystalloid perfusion fluid, which not only affords oxygen-carrying capacity and coronary flow autoregulation within the physiologic range but also provides biochemical functions, such as buffering of oxygen radicals and catabolism of nitric oxide (NO).

\section{RKI}

RKIs are beneficial in organ transplantation. Shiotani and colleagues ${ }^{17}$ demonstrated that RKI reduced acute hepatocellular necrosis and apoptosis induced by cold ischemiareperfusion injury in a rat model of liver transplantation. 

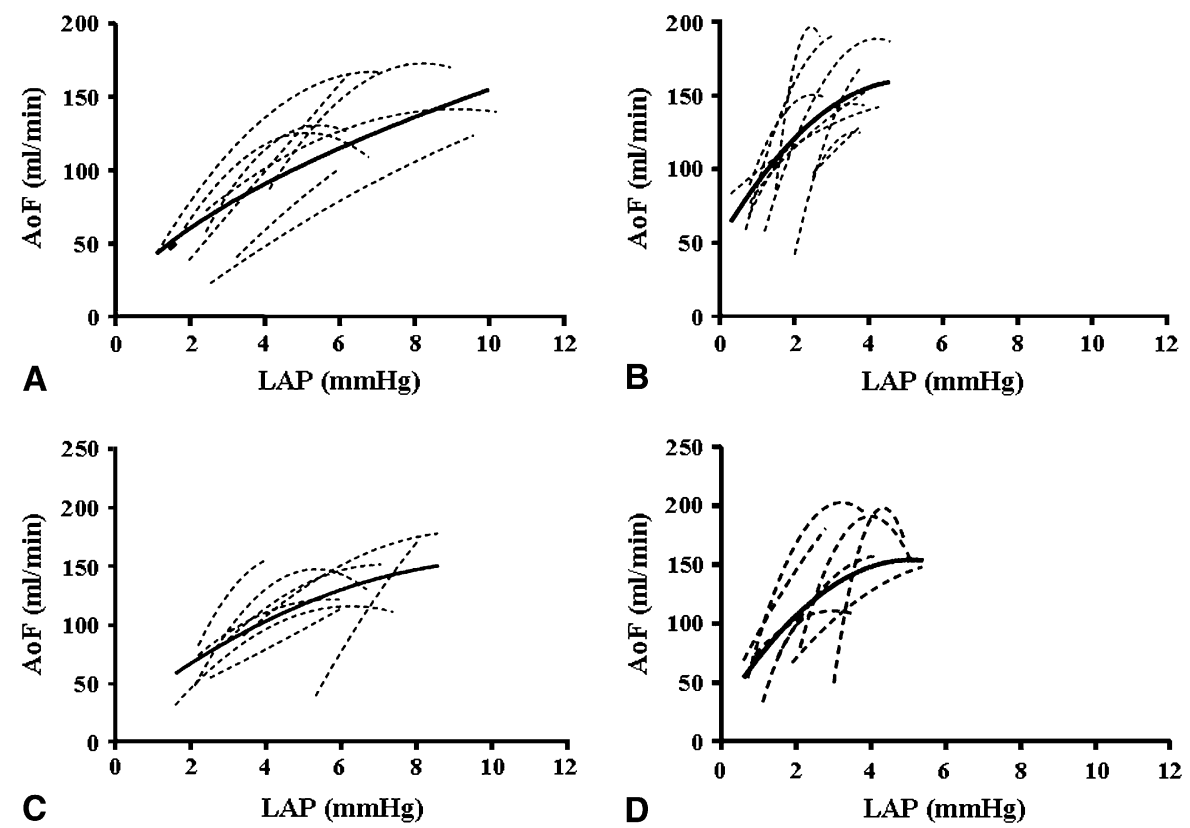

FIGURE 1. Modified Frank-Starling curves obtained by means of multiple regression analysis with dummy variables in each group. The black broken lines show the individual curves in each group. The black solid lines represent the regression equations for each curve. Representative equations are as follows: (A) $y=-2.01 x^{2}+36.75 x-19.85$ at 60 minutes in the control group; (B) $y=-5.91 x^{2}+62.08 x+18.13$ at 60 minutes in the Rho-kinase inhibitor group; (C) $\mathrm{y}=-0.16 \mathrm{x}^{2}+19.05 \mathrm{x}+27.24$ at 120 minutes in the control group; (D) $\mathrm{y}=-5.49 \mathrm{x}^{2}+63.44 \mathrm{x}-3.74$ at 120 minutes in the Rho-kinase inhibitor group. The values of the $\mathrm{x}$-intercept and the slope at the $\mathrm{x}$-intercept obtained from individual curves in each group are shown in Table 1. AoF, Aortic flow; $L A P$, left atrial pressure.

Hattori and associates ${ }^{9}$ described that long-term treatment with RKI suppressed cardiac allograft vasculopathy in mice. The influence of Rho-kinase during cardiac allograft storage is not clear.

The report by Yada and coworkers ${ }^{10}$ showed that RKI supplanted directly to the coronary artery dose-dependently caused vasodilatation and increased CBF in a canine model. The most effective dose of RKI was $0.1 \mathrm{mg} / \mathrm{kg}$. Thus we used that dose of RKI in the supplanted solution directly. Our preliminary experiment of $1 \mathrm{mg} / \mathrm{kg}$ RKI supplanted into the cardioplegic solution did not improve cardiac recovery. Moreover, RKI added to the support rabbit during reperfusion produced profound systemic vasodilation and instability. Therefore we assessed RKI during harvest and allograft storage to minimize changes in the systemic pressure of the support rabbit.

Inokuchi and colleagues ${ }^{18}$ reported that fasudil $(1.5 \mathrm{mg} /$ $\mathrm{kg}$ ) administered into intractable spastic arteries was effec-

TABLE 1. Values of the $x$-intercept and the slope at the $x$-intercept in each group

\begin{tabular}{lccccc}
\hline & \multicolumn{2}{c}{$\mathbf{6 0}$ min } & & \multicolumn{2}{c}{$\mathbf{1 2 0}$ min } \\
\cline { 2 - 3 } \cline { 5 - 6 } Group & $\mathbf{x}$-Intercept & Slope & & x-Intercept & Slope \\
\hline Control & $0.59 \pm 0.77$ & $34.39 \pm 3.09$ & & $-1.40 \pm 1.26$ & $42.39 \pm 5.06$ \\
RKI & $-0.28 \pm 0.30$ & $65.38 \pm 3.49$ & & $0.08 \pm 0.50$ & $61.15 \pm 5.87$ \\
$P$ value & .017 & $<.01$ & .013 & $<.01$ \\
\hline
\end{tabular}

RKI, Rho-kinase inhibitor. tive in a clinical setting and showed few systemic adverse effects. Species differences could account for these different dosing needs.

Fasudil hydrochloride and Y27632 are available RKIs. We chose the former because it is already available in clinical settings. ${ }^{4,5}$ The latter is used only in animal studies because of its metabolic byproducts.

\section{Enhanced Cardiac Function Recovery}

Allograft contractile performance was evaluated with modified Frank-Starling curves. RKI enhanced the recovery of $\mathrm{LV}$ function after reperfusion. The curve at 60 minutes in the RKI group was close to that of a normal heart, as was shown in our previous study. ${ }^{12}$ After ischemia and reperfusion, intracellular Rho-kinase is induced by angiotensin II, thrombin, and endothelin I. Antagonists or inhibitors for those agonists usually attenuate ischemia-reperfusion injury, ${ }^{3}$ with efficacy similar to that of RKI, indicating that RKI influences multiple signaling pathways.

In the RKI group increased CBF might result from preserved endothelial function and coronary vasodilatation. Improved endothelial function could result in enhanced contractile function by improving myocardial perfusion and a washout of toxic metabolites that accumulate during storage. RKI treatment slightly increased CKMB levels at 120 minutes but did not change necrosis or myocardial structure under hematoxylin and eosin staining and did not affect 

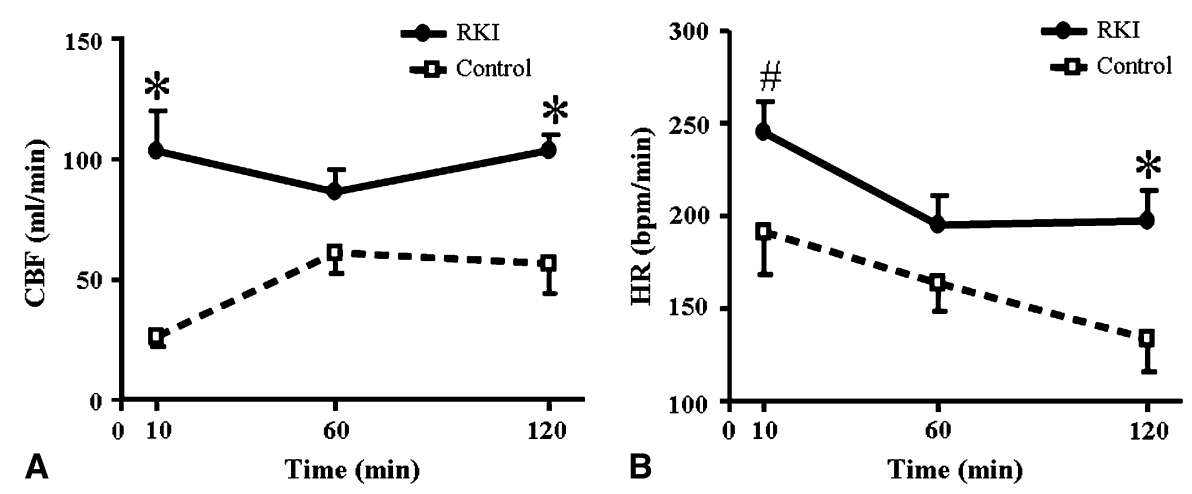

FIGURE 2. A, Maximum coronary blood flow $(C B F)$ after reperfusion. The CBF rate was higher in the Rho-kinase inhibitor (RKI) group than in the control group at both 10 and 120 minutes $(* P<.05)$. B, Heart rate $(H R)$ of the donor in each group after reperfusion. The HR in the RKI group was more similar to normal levels compared with that in the control group. HR at 120 minutes was significantly higher in the RKI group than in the control group $\left({ }^{*} P<.05\right.$, $\left.\# P=.08\right)$.

apoptosis. Such changes would presumably appear later because the allograft was appropriately preserved and incidental apoptosis in ischemia-reperfusion injury develops during late reperfusion. ${ }^{19}$

\section{Preserved Endothelial Function}

Postischemic endothelial dysfunction occurs in several models of cardiac injury. ${ }^{20}$ The primary consequence of impaired endothelial function is the reduction of $\mathrm{NO}$
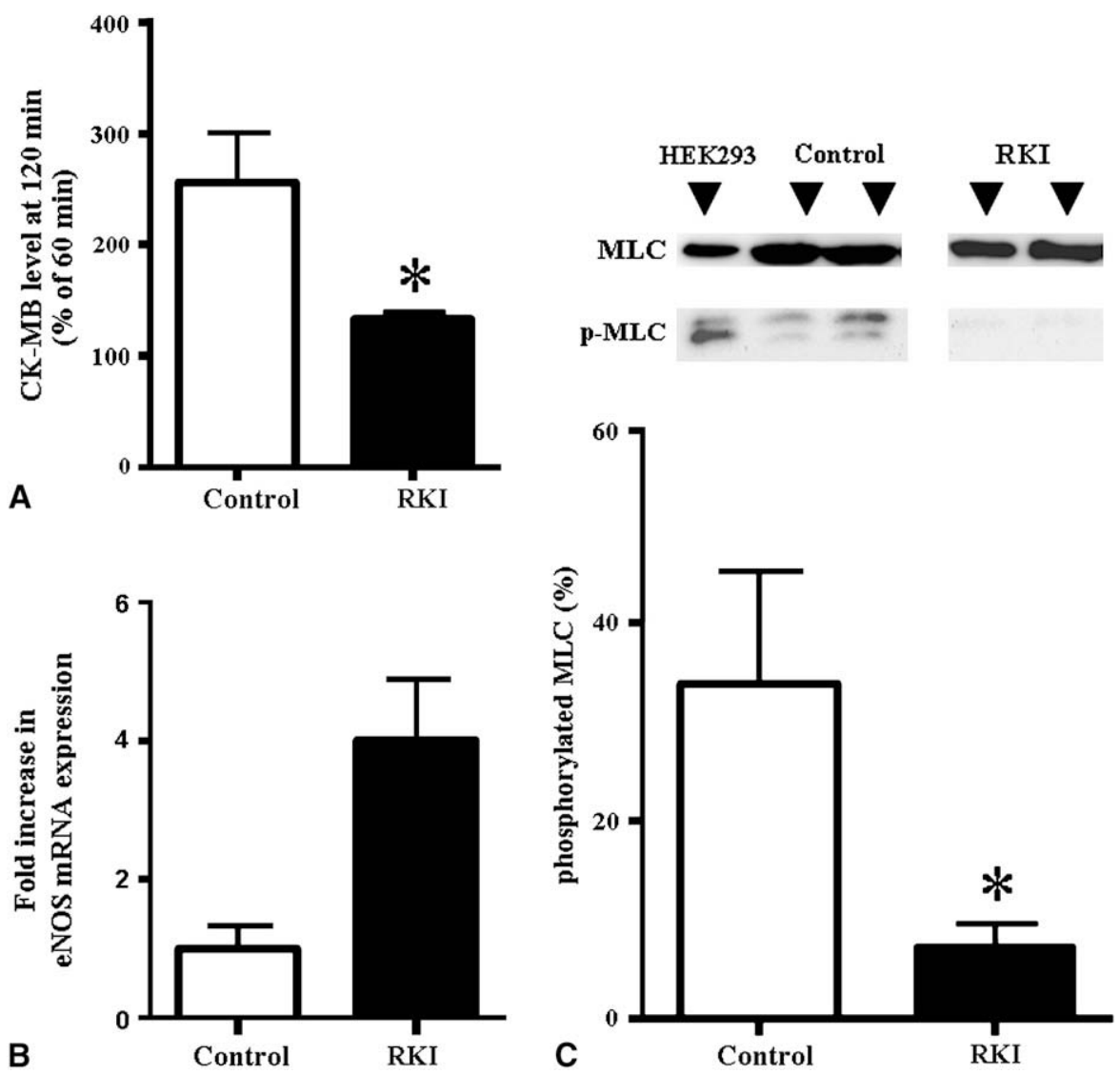

FIGURE 3. A, Comparison of creatine kinase-MB (CK-MB) levels at 120 minutes with those at 60 minutes. Rate of change in CK-MB level in the control group was $256.19 \% \pm 125.15 \%$, whereas that in the Rho-kinase inhibitor (RKI) group was $132.89 \% \pm 17.40 \%(* P<.05)$. B, Relative expression of endothelial nitric oxide synthase (eNOS) mRNA in the control and RKI groups. eNOS expression in the RKI group was 4-fold higher than that in the control group. C, Suppression of phosphorylated myosin light chain (p-MLC) by RKI. The extent of p-MLC was significantly inhibited in the RKI group compared with that in the control group $(33.82 \% \pm 11.37 \%$ vs $7.26 \% \pm 2.31 \%, * P<.05)$. 
production released by endothelial cells. NO is synthesized from L-arginine by NO synthase and modulates vascular tone, attenuates platelet aggregation and neutrophil function, ${ }^{21}$ and reduces free radical damage. ${ }^{22} \mathrm{NO}$ synthesis inhibitors and eNOS-deficient mice increase infarct size after ischemia and reperfusion. ${ }^{23}$ Therefore restored NO synthesis is mandatory for appropriate cardiac performance. RKI exerts cardioprotective effects on coronary ischemia-reperfusion injury in vivo, possibly by preserving eNOS expression. ${ }^{24}$ The overexpression of a dominant-negative mutant of Rho A in cultured endothelial cells upregulates the expression of eNOS mRNA, and the activation of Rho A downregulates eNOS mRNA. ${ }^{25}$ Inhibition of Rho-kinase signaling helps preserve eNOS mRNA levels.

NO production can be evaluated by measuring the amount of NO in serum samples, Western blotting for eNOS, and detection of eNOS mRNA by means of polymerase chain reaction, but we prefer real-time reverse transcriptasepolymerase chain reaction. RKI treatment increased eNOS mRNA 4-fold compared with that seen in the control group. RKI could improve cardiac function through NO-dependent endothelial protection after ischemia and reperfusion.

\section{Analysis of RKI Activity}

We added RKI to all solutions, including the cardioplegic solution, UW solution, and storage solution. However, it is unclear whether the chemical can penetrate cells at a low temperature. To evaluate the bioactivity of Rho A and Rho-kinase, phosphorylation of the $\alpha$-adducin, ezrin, radixin, and moesin family and the myosin-binding subunit of myosin phosphatase, as well as expression of Rho A are usually measured. We used an anti-p-MLC antibody that is specific to MLC in the endothelium and vascular smooth muscle but not cardiomyocytes. RKI reduced p-MLC levels in the allograft for at least 2 hours after reperfusion. RKI is a vasorelaxant in the endothelium and vascular smooth muscle in vivo and in vitro. ${ }^{26,27}$

\section{LIMITATIONS}

Our data are limited to the acute setting after reperfusion. Although a combination of early endothelial and myocardial protection will presumably provide long-term benefits, further chronic RKI treatment is required to test this. Rho and Rho-kinase activation is difficult to measure in rabbits because of limited antibody selection.

Although the transition of the donor heart rate in each group after reperfusion was in direct proportion to $\mathrm{CBF}$, the relationships were inversely proportional. At 60 minutes, heart rate in the RKI group decreased but was increased in the control group. We are unable to explain the reason for this difference.

Coronary vasculopathy after cardiac transplantation is an important cause of poor outcomes after cardiac transplantation, including reduced survival. ${ }^{28}$ Improvement of both endothelial and myocardial protection during allograft storage might improve early and long-term outcomes. These results might have clinical relevance for the prevention of low coronary flow phenomenon and postoperative low output syndrome induced by prolonged cardiac ischemia.

\section{CONCLUSIONS}

With a working model using cross-circulated blood perfusion in the rabbit heart, we showed that RKI during allograft harvest and storage enhanced CBF and ventricular recovery through NO-dependent endothelial protection after reperfusion. Rho and Rho-kinase are implicated in endothelial dysfunction induced by means of ischemia and reperfusion. These results indicate that RKI could be a potential therapy for preventing early myocardial dysfunction after transplantation.

We thank Tomomi Yamada, MS, for statistical analysis; Miho Miyakawa and Eiko Iwata for pathologic technical advice and assistance; and Kensuke Egashira, MD, PhD, Department of Cardiovascular Medicine, for pathologic experimental support.

\section{References}

1. Taylor DO, Edwards LB, Boucek MM, Trulock EP, Keck BM, Hertz MI. The registry of the international society for heart lung transplantation: twenty-first official adult heart transplant report-2004. J Heart Lung Transplant. 2004;23:796-803.

2. Russo JM, Chen JM, Sorabella RA, Martens TP, Garrido M, Davis RR, et al. The effect of ischemic time on survival after heart transplantation varies by donor age: an analysis of the united network for organ sharing database. J Thorac Cardiovasc Surg. 2007;133:554-9.

3. Simokawa H. Rho-kinase as a novel therapeutic target in treatment of cardiovascular disease. J Cardiovasc Pharmacol. 2002;39:319-27.

4. Shibuya M, Suzuki Y, Suguta K, Saito I, Sasaki T, Takakura K, et al. Effect of AT877 on cerebral vasospasm after aneurismal subarachnoid hemorrhage: results of a protective placebo-controlled double-blind trial. J Neurosurg. 1992;76: 571-7.

5. Shimokawa H, Hiramori K, Iinuma H, Hosoda S, Kishida H, Osada H, et al. Antianginal effect of fasudil, a Rho-kinase inhibitor, in patients with stable angina: a multicenter study. J Cardiovasc Pharmacol. 2002;40:751-61.

6. Narumiya S. The small GTPase Rho: cellular signal function of signal transduction. J Biochem (Tokyo). 1996;120:215-28.

7. Amano M, Chihara K, Kimura K, Fukata Y, Nakamura N, Matsumura Y, et al. Formation of actin stress fibers and focal adhesions enhanced by Rho-kinase. Science. 1997;275:1308-11.

8. Horwitz AR, Parsons JT. Cell migration: movin' on. Science. 1999;286:1102-3.

9. Hattori T, Shimokawa H, Higashi M, Hiroki J, Mukai Y, Tsutsui H, et al. Longterm inhibition of Rho-kinase suppresses left ventricular remodeling after myocardial infarction in mice. Circulation. 2004;109:2234-9.

10. Yada T, Shimokawa H, Hiramatsu O, Kajita T, Shigeto F, Tanaka E, et al. Beneficial effect of hydroxyfasudil, a specific Rho-kinase inhibitor, on ischemia/reperfusion injury in canine coronary microcirculation in vivo. $J$ Am Coll Cardiol. 2005;45:599-607.

11. Kajihara N, Morita S, Tanoue Y, Boku N, Eto M, Nishida T, et al. The UW solution has greater potential for longer preservation periods than the Cersior solution: comparative study for ventricular and coronary endothelial function after 24-h heart preservation. Eur J Cardiothorac Surg. 2006;29:784-9.

12. Kajihara T, Morita S, Nishida T, Tatewaki H, Eto M, Egashira K, et al. Transfection with a dominant-negative inhibitor of myocyte chemoattractant protein-1 gene improves cardiac function after 6 hours of cold preservation. Circulation. 2003;108(suppl II):II213-8.

13. Boku N, Tanoue N, Kajihara N, Eto M, Masuda M, Morita S. A comparative study of cardiac preservation with Cersior or university of Wisconsin solution with or without prior administration of cardioplegia. J Heart Lung Transplant. 2006 25:219-25. 
14. Tanoue Y, Morita S, Hisahara M, Tominaga R, Kawachi Y, Yasui H. Arresting donor hearts with extracellular-type cardioplegia prevents vasoconstriction induced by UW solution. Cardiovasc Surg. 1998;6:622-8.

15. Hallam KM, Edirisinghe I, Balasuriya UBR, Gunawardena S, Bravo EM, Ravi K, et al. Effects of mitral regurgitation on the reflex diuresis to pulmonary lymphatic obstruction in rabbits. Exp Lung Res. 2007;33:259-75.

16. Furuyama T, Komori K, Shimokawa H, Matsumoto Y, Uwatoku T, Hirano K, et al. Long-term inhibition of Rho kinase suppresses internal thickening in autologous vein grafts in rabbits. J Cardiovasc Surg. 2006;43:1249-55.

17. Shiotani S, Shimoda M, Suehiro T, Soejima Y, Yosizumi T, Shimokawa H, et al. Involvement of Rho-kinase in cold ischemia-reperfusion injury after liver transplantation in rats. Transplantation. 2004;78:375-82.

18. Inokuchi K, Ito A, Fukumoto Y, Matoba T, Shiose A, Nisida T, et al. Usefulness of fasudil, a Rho-kinase inhibitor, to treat intractable severe coronary spasm after coronary artery bypass surgery. J Cardiovasc Pharmacol. 2004;44:275-7.

19. Zhao Z, Velez DA, Wang N, Hewan-Lowe KO, Nakamura M, Guyton RA, et al. Progressively developed myocardial apoptotic cell death during late phase of reperfusion. Apoptosis. 2001;6:279-90.

20. Lefer AM, Tsao PS, Lefer DJ, Ma XL. Role of endothelial dysfunction in the pathogenesis of reperfusion injury after myocardial ischemia. FASEB J. 1991;261: 2029-34.
21. Kubes P, Suzuki M, Granger DN. Nitric oxide: an endogenous modular of leukocyte adhesion. Proc Natl Acad Sci U S A. 1991;88:4651-5.

22. Gryglewski RJ, Palmer RMJ, Moncada S. Superoxide anion is involved in the breakdown of endothelium-derived relaxing factor. Nature. 1986;320:454-6.

23. Jones SP, Girod WG, Palazzo AJ, Granger DN, Grisham MB, Jourd'Heuil D, et al. Myocardial ischemia-reperfusion injury is exacerbated in the absence of endothelial cell nitric oxide synthase. Am J Physiol Heart Circ Physiol. 1999;276:H1567-73.

24. Bao W, Hu E, Boyce R, Mirabile R, Thudium TD, Ma X, et al. Inhibition of Rhokinase protects the heart against ischemia/reperfusion injury. Cardiovasc Res. 2004;61:548-58.

25. Laufs U, Liao JK. Post-transcriptional regulation of endothelial nitric oxide synthase mRNA stability by Rho GTPase. J Biol Chem. 1998;273:24266-71.

26. Asano T, Ikegaki I, Satoh S, Suzuki Y, Shibuya M, Takayasu M, et al. Mechanism of action of a novel antivasospasm drug, HA1077. J Pharmacol Exp Ther. 1987; 241:1033-40.

27. Asano T, Suzuki T, Tsuchiya M, Satoh S, Ikegaki I, Shibuya M, et al. Vasodilator actions of HA1077 in vitro and in vivo putatively mediated by the inhibition of protein kinase. Br J Pharmacol. 1989;98:1091-100.

28. Hollenberg SM, Klein LW, Parrillo JE, Scherer M, Burns D, Tamburro P, et al. Coronary endothelial dysfunction after heart transplantation predicts allograft vasculopathy and cardiac death. Circulation. 2001;104:3091-4. 